\section{Biosimilar development and regulation in Japan}

In Japan, biosimilars guidelines following the principles of
the EU framework were established by Japan's Ministry of Health, Labor and Welfare in March 2009. The guidelines cover the manufacturing process, characterization of quality attributes, and clinical and non-clinical studies for biosimilars. In this paper, the requirements for regulatory approval of biosimilars in Japan are outlined.

Keywords: Biosimilar, biotechnological drug, Japan, regulatory

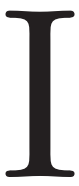
n Japan, a biosimilar product is defined as a biotechnological drug product developed by a different company, which is comparable to an approved biotechnology-derived product (reference product) of an innovator company $[1,2]$.

Biosimilars may be recombinant proteins and polypeptide products, their derivatives, and products of which they are components, e.g. conjugates. Biosimilars are proteins and polypeptide products produced from recombinant expression systems using micro-organisms or cultured cells and that can be highly purified and well-characterized using an appropriate set of analytical procedures.

A biosimilar product can generally be developed on the basis of data that demonstrates the comparability between the biosimilar product and the reference product with respect to quality, safety and efficacy, or other relevant data, see Figure 1.

The reference products should be drugs approved in Japan and be the same product throughout the development period of the biosimilar products.

\section{Manufacturing process}

It should be established that the manufacturing process for the biosimilar is highly consistent and robust. If the host cell line used for the production of the reference product is disclosed by the innovator company, it is preferred that the same cell line is used. The cell banks should be established and characterized following ICH Q5A, Q5B and Q5D guidelines. The manufacturing processes should be adapted to improve the safety of the biosimilar, as long as this does not affect efficacy [1].

\section{Quality characterization}

The biosimilar should be fully characterized using state of the art analytical methods, and should include:

1) structural characterization

2) physicochemical properties

3) biological activity

4) immunological properties

5) impurities

\section{Formulation}

The dosage form and route of administration of the biosimilar product should be the same as that of the reference product. However, it is not essential for the biosimilar product to have the same formulation as the reference product, provided that the safety and efficacy are not affected.
Stability

Long-term, real-time, real-condition stability studies following the ICH Q5C guideline are required. The expiry date for the biosimilar product should be determined based on data from real-time/real-temperature studies. A comparison of stability with the reference product will not necessarily be required. Accelerated and stress stability studies are recommended to obtain useful data for evaluating the properties of the biosimilar product.

Comparability studies on quality attributes

In addition to elucidating the quality attributes of the biosimilar, comparability exercises comparing quality attributes between the biosimilar and the reference product should be conducted [1].

\section{Non-clinical studies}

Non-clinical studies that can ensure the safety for administration to humans should be performed and completed prior to initiation of clinical studies.

Both comparability and individual assessments are applicable, depending on the purpose of the study.

\section{Pharmacokinetics}

It is useful to compare the non-clinical pharmacokinetics in some glycoproteins.

\section{Pharmacological studies}

Comparability of the pharmacological action should be directly evaluated.

\section{Toxicity studies}

- Repeated-dose toxicity studies may be useful to evaluate both single-dose and repeated-dose toxicity.

Figure 1: Dossier requirements for originator biologicals and biosimilars in Japan [1, 2]

$\begin{array}{lll}\begin{array}{l}\text { Dossiers of the originator } \\ \text { (reference) product }\end{array} & \begin{array}{l}\text { Dossiers of the } \\ \text { biosimilar product }\end{array} & \text { Comment }\end{array}$

(reference) product biosimilar product
Manufacturing process

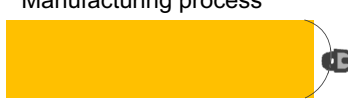

Characterization of quality attributes

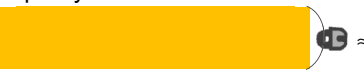

$D \approx$ quality attributes

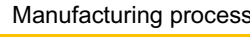

$\neq$

Non-clinical study

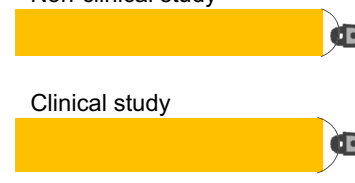

\section{Non-clinical study}

$\approx$

Clinical study
To establish a stable and robust manufacturing process

To analyze the quality attributes individually

Comparability study

Individual study

nformation 
- Local tolerance could be evaluated - in repeated-dose toxicity studies.

- A direct comparative study of the toxicity profile may not always be necessary, e.g. for process-related impurities.

- Other general non-clinical safety studies are generally unnecessary.

\section{Clinical studies}

Where the data from quality characterization and non-clinical studies is insufficient to demonstrate comparability with the reference product clinical studies are required. Clinical studies should be designed based on the data from quality characterization, non-clinical studies and comparability studies [1].
It is necessary to carry out comparability studies between the biosimilar and the reference product for pharmacokinetic, pharmacodynamic, efficacy and safety parameters.

Where comparability has been demonstrated through clinical pharmacokinetic, pharmacodynamic or pharmacokinetic/pharmacodynamic studies, further clinical studies may be reduced in some cases. In general, a well-designed crossover study should be considered in order to evaluate the comparability between the biosimilar and the reference product. However, a crossover study may not be suitable for long halflife products or products which may cause formation of antibodies.

\section{Competing interests: None.}

Provenance and peer review: Article prepared based on extensive research, internally peer reviewed.

Michelle Derbyshire, PhD, GaBI Online Editor

\section{References}

1. Arato T. Recent regulations of biosimilars in Japan. Pharmaceuticals and Medical Devices Agency. 47th Annual Meeting, DIA 2011; 2011 Jun 19-23, Chicago, USA.

2. Arato T. Regulatory guidelines for biosimilars in Japan. 10th EGA International Symposium on Biosimilar Medicines; 2012 April 20; London, UK. DOI: 10.5639/gabij.2013.0204.055

Copyright (c) 2013 Pro Pharma Communications International 